\title{
Relativistic Correction to Charmonium Dissociation Temperature
}

\author{
Xingyu Guo, Shuzhe Shi, and Pengfei Zhuang \\ Physics Department, Tsinghua University, Beijing 100084, China
}

(Dated: November 18, 2018)

\begin{abstract}
By solving the covariant relativistic Schrödinger equations for a pair of heavy quarks, we obtained the wave functions for the ground and excited quarkonium states at finite temperature. In comparison with the non-relativistic calculation, the $J / \psi$ dissociation temperature determined by the infinity size or zero binding energy of the system increases $7 \%-13 \%$, when the central potential varies between the free energy and internal energy.
\end{abstract}

It is widely accepted that there exists a quantum chromodynamics (QCD) phase transition from hadron gas to a new state of matter, the quark-gluon plasma (QGP) at finite temperature and baryon density. To probe the realization of such a phase transition in relativistic heavy ion collisions, some signatures of the new state of matter have been discussed for decades [1], and among which the quarkonium suppression is considered as a smoking gun of the formation of QGP 2].

A quarkonium is a deeply bound state of a pair of heavy quarks, its dissociation temperature $T_{D}$ in a hot medium should be higher than the critical temperature $T_{c}$ for the deconfinement of light hadrons. Since a quarkonium is so heavy, one normally uses non-relativistic Schrödinger equation to describe its dynamical evolution at finite temperature. The free energy $F$ between a pair of heavy quarks can be extracted from the lattice QCD simulations $[3$, 44. Taking $F$ and the internal energy $U$ as the two limits of the heavy quark potential $V$, which correspond respectively to a slow and a rapid quarkonium dissociation in the hot medium, the $J / \psi$ dissociation temperature $T_{D}$ determined by the infinity size or the zero binding energy of the system is in between $1.2 T_{c}$ and $2 T_{c}[3,5]$.

A nature question we ask ourselves is the relativistic correction to the dynamical evolution of a quarkonium in the hot medium. The correction to a bottonium is expected to be neglected safely, but for a lighter quarkonium like $J / \psi$, the correction might be remarkable. The two-body Dirac equation (TBDE) 6 [1] of constrained dynamics was successfully applied to the relativistic description of light meson spectra 12 17] in vacuum. In this Letter we take the TBDE to calculate the charmonium wave functions at finite temperature and see the relativistic correction to the dissociation temperature.

We can qualitatively estimate the relativistic effect on the quarkonium dissociation before a strict calculation. Neglecting the quark spin, the relative part of the Hamiltonian for a pair of heavy quarks can be approximately written as a non-relativistic form

$$
H=\sqrt{\mu^{2}+p^{2}}-\mu+V(r) \simeq \frac{p^{2}}{2 \mu}+V_{e f f}
$$

with an effective potential

$$
V_{e f f}=V-\frac{p^{4}}{8 \mu^{3}},
$$

where $\mu=m_{Q} / 2$ is the reduced mass with $m_{Q}$ being the heavy quark mass. Since the relativistic correction leads to a deeper potential well, $V_{\text {eff }}<V$, the quarkonium becomes a more deeply bound state and the temperature needed to dissociate the quarkonium should be higher.

We now calculate the wave functions for a pair of heavy quarks in the frame of TBDE. Taking Pauli reduction and scale transformation 18], the Dirac equation can be expressed as a covariant relativistic Schrödinger equation for a four-component spinor [10]. Explicitly, the radial motion relative to the center of mass is controlled by the following four equations [13],

$$
\begin{aligned}
& {\left[-\frac{d^{2}}{d r^{2}}+\frac{J(J+1)}{r^{2}}+2 m_{w} B+B^{2}-A^{2}+2 \epsilon_{w} A+\Phi_{D}-2 \Phi_{S O}+\Phi_{S S}+2 \Phi_{T}-2 \Phi_{S O T}\right] u_{1}^{0}=b^{2} u_{1}^{0}, } \\
& {\left[-\frac{d^{2}}{d r^{2}}+\frac{J(J-1)}{r^{2}}+2 m_{w} B+B^{2}-A^{2}+2 \epsilon_{w} A+\Phi_{D}+2(J-1) \Phi_{S O}+\Phi_{S S}+\frac{2(J-1)}{2 J+1}\left(\Phi_{S O T}-\Phi_{T}\right)\right] u_{1}^{+} } \\
+ & \frac{2 \sqrt{J(J+1)}}{2 J+1}\left(3 \Phi_{T}-2(J+2) \Phi_{S O T}\right) u_{1}^{-}=b^{2} u_{1}^{+}, \\
& {\left[-\frac{d^{2}}{d r^{2}}+\frac{(J+1)(J+2)}{r^{2}}+2 m_{w} B+B^{2}-A^{2}+2 \epsilon_{w} A+\Phi_{D}-2(J+2) \Phi_{S O}+\Phi_{S S}+\frac{2(J+2)}{2 J+1}\left(\Phi_{S O T}-\Phi_{T}\right)\right] u_{1}^{-} } \\
+ & \frac{2 \sqrt{J(J+1)}}{2 J+1}\left(3 \Phi_{T}+2(J-1) \Phi_{S O T}\right) u_{1}^{+}=b^{2} u_{1}^{-}
\end{aligned}
$$


for the spin triplet $u_{1}^{0}, u_{1}^{+}$and $u_{1}^{-}$with quantum numbers $n^{2 s+1} L_{J}=n^{3} L_{L}, n^{3} L_{L+1}$ and $n^{3} L_{L-1}$, and

$$
\left[-\frac{d^{2}}{d r^{2}}+\frac{J(J+1)}{r^{2}}+2 m_{w} B+B^{2}-A^{2}+2 \epsilon_{w} A+\Phi_{D}-3 \Phi_{S S}\right] u_{0}=b^{2} u_{0}
$$

for the spin singlet $u_{0}$ with quantum numbers $n^{1} L_{L}$, where $b^{2}=\left(m_{m}^{2}-4 m_{Q}^{2}\right) / 4$ is the energy eigenvalue in the meson rest frame with $m_{m}$ being the meson mass, $n$ is the principal quantum number, and $L, s$ and $J$ are the orbital, spin and total angular momentum numbers. Note that the components $u_{1}^{+}$with $J=L+1$ and $u_{1}^{-}$with $J=L-1$ are coupled to each other. Following the notations in [13], we have separated the central potential into two parts,

$$
V(r)=A(r)+B(r),
$$

and the abbreviations for the Darwin, spin-spin, spin-orbit and tensor terms introduced in the dynamical equations are defined as [13]

$$
\begin{aligned}
\Phi_{D} & =M+F^{2}+K^{\prime 2}-\nabla^{2} F+2 K^{\prime} P-2\left(F^{\prime}+\frac{1}{r}\right) Q \\
\Phi_{T} & =\frac{1}{3}\left[N+2 F^{\prime} K^{\prime}-\nabla^{2} K+\left(3 F^{\prime}-K^{\prime}+\frac{3}{r}\right) P+\left(F^{\prime}-3 K^{\prime}+\frac{1}{r}\right) Q\right] \\
\Phi_{S O} & =-\frac{F^{\prime}}{r}+K^{\prime} P-\left(F^{\prime}+\frac{1}{r}\right) Q \\
\Phi_{S S} & =O+\frac{2}{3} F^{\prime} K^{\prime}-\frac{1}{3} \nabla^{2} K+\frac{2}{3} K^{\prime} P-2\left(F^{\prime}+\frac{1}{3 r}\right) Q \\
\Phi_{S O T} & =-\frac{K^{\prime}}{r}+\left(F^{\prime}+\frac{1}{r}\right) P-K^{\prime} Q \\
F & =\frac{1}{2} L-\frac{3}{2} G, \\
G & =-\frac{1}{2} \ln \left(1-2 \frac{A}{m_{m}}\right), \\
K & =\frac{1}{2} L+\frac{1}{2} G, \\
L & =\ln \sqrt{1+\frac{2 m_{w} B+B^{2}}{m_{Q}^{2}\left(1-2 A / m_{m}\right)}}, \\
M & =-\frac{1}{2} \nabla^{2} G+\frac{3}{4} G^{2}+G^{\prime} F^{\prime}-K^{\prime 2} \\
N & =\frac{1}{3}\left[\nabla^{2} K-\frac{1}{2} \nabla^{2} G+\frac{3}{2} \frac{G^{\prime}-2 K^{\prime}}{r}+F^{\prime}\left(G^{\prime}-2 K^{\prime}\right)\right] \\
O & =\frac{1}{3} \nabla^{2}(K+G)-\frac{1}{3} F^{\prime}\left(G^{\prime}+K^{\prime}\right)-\frac{1}{2} G^{\prime 2}, \\
P & =\frac{\sinh 2 K}{r}, \\
Q & =\frac{\cosh 2 K-1}{r}
\end{aligned}
$$

and $m_{w}=m_{Q}^{2} / m_{m}, \epsilon_{w}=\left(m_{m}^{2}-2 m_{Q}^{2}\right) /\left(2 m_{m}\right), F^{\prime}=d F / d r, G^{\prime}=d G / d r$ and $K^{\prime}=d K / d r$.

We now focus on the charmonium states. The $J / \psi$ state includes two components $1^{3} S_{1}$ and $1^{3} D_{1}$ determined simultaneously by the two coupled equations of (3) with energy eigenvalue $b^{2}=\left(m_{J / \psi}^{2}-4 m_{c}^{2}\right) / 4$. Similarly, the two components $2^{3} S_{1}$ and $2^{3} D_{1}$ for the state $\psi^{\prime}$ are controlled by the two coupled equations but with eigenvalue $b^{2}=\left(m_{\psi^{\prime}}^{2}-4 m_{c}^{2}\right) / 4$. The $\chi_{0}$ state $1^{3} P_{0}$ is described by the last equation of (3) with disappeared component $u_{1}^{+}$, and the $\chi_{1}$ state $1^{3} P_{1}$ is characterized by the independent equation (4) for $u_{1}^{0}$.

In vacuum, the potential between two quarks is usually taken as the Cornell form, including a Coulomb-like part which dominates the wave functions around $r=0$ and a linear part which leads to the quark confinement,

$$
\begin{aligned}
& A(r)=-\frac{\alpha}{r}, \\
& B(r)=\sigma r .
\end{aligned}
$$


The three parameters in the model, namely the charm quark mass $m_{c}$ in the Schrödinger equation and the two coupling constants $\alpha$ and $\sigma$ in the potential, can be fixed by fitting the charmonium masses in vacuum. By taking $m_{c}=1.422 \mathrm{GeV}, \alpha=0.492$ and $\sigma=0.186 \mathrm{GeV}^{2}$, we obtain the charmonium masses $m_{J / \psi}=3.113 \mathrm{GeV}, m_{\psi^{\prime}}=3.692$ $\mathrm{GeV}, m_{\chi_{0}}=3.404 \mathrm{GeV}$ and $m_{\chi_{1}}=3.504 \mathrm{GeV}$, which are very close to the experimental values $m_{J / \psi}=3.097 \mathrm{GeV}$, $m_{\psi^{\prime}}=3.686 \mathrm{GeV}, m_{\chi_{0}}=3.415 \mathrm{GeV}$ and $m_{\chi_{1}}=3.511 \mathrm{GeV}$.

The charmonium dissociation temperature in a hot medium can be determined by solving the corresponding dynamical equation for the $c \bar{c}$ system with potential $V$ between the two heavy quarks at finite temperature. The potential depends on the dissociation process in the medium. At the moment, we know only its two limits. For a rapid dissociation where there is no heat exchange between the heavy quarks and the medium, the potential is just the internal energy $U$, while for a slow dissociation, there is enough time for the heavy quarks to exchange heat with the medium, the free energy $F$ which can be extracted from the lattice calculations is taken as the potential[19]. From the thermodynamic relation $F=U-T \mathcal{S}$ where $\mathcal{S}$ is the entropy, the potential well is deeper for $V=U$ and therefore the dissociation temperature of charmonium states with potential $V=U$ is higher than that with $V=F$. In the literatures, a number of effective potentials in between $F$ and $U$ have been used to evaluate the charmonium evolution in QCD medium[3, [5, 19, 20]. In non-relativistic case, H.Satz and his collaborators [3, [5] solved the Schrödinger equation and found the dissociation temperatures $T_{d} / T_{c}=2.1$ and 1.26 for $V=U$ and $V=F$ respectively, where $T_{c}=165 \mathrm{MeV}[\underline{3}, 5]$ is the critical temperature for the deconfinement.

Considering the Debye screening at finite temperature, the potential $V=F=A+B$ can be written as $[\underline{3},[5]$

$$
\begin{aligned}
& A(r, T)=-\frac{\alpha}{r} e^{-\mu r}, \\
& B(r, T)=\frac{\sigma}{\mu}\left[\frac{\Gamma\left(\frac{1}{4}\right)}{2^{\frac{3}{2}} \Gamma\left(\frac{3}{4}\right)}-\frac{\sqrt{\mu r}}{2^{\frac{3}{4}} \Gamma\left(\frac{3}{4}\right)} K_{\frac{1}{4}}\left(\mu^{2} r^{2}\right)\right]-\alpha \mu,
\end{aligned}
$$

where $\Gamma$ is the Gamma function, $K$ is the modified Bessel function of the second kind, and the temperature dependent parameter $\mu(T)$, namely the screening mass or the inverse screening radius, can be extracted from fitting the lattice simulated free energy [3, 4]. From the known free energy $F$, one can then obtain the other limit of the potential, $V=U=F+T \mathcal{S}$ by taking $\mathcal{S}=-\partial F / \partial T$.

We use the inverse power method 21] to solve the Schrödinger equations (3) and (4) and obtain the radial wave functions

$$
\Psi(r, T)=\frac{u(r, T)}{r}
$$

for the charmonium states $J / \psi, \psi^{\prime}$ and $\chi_{c}$ at finite temperature.

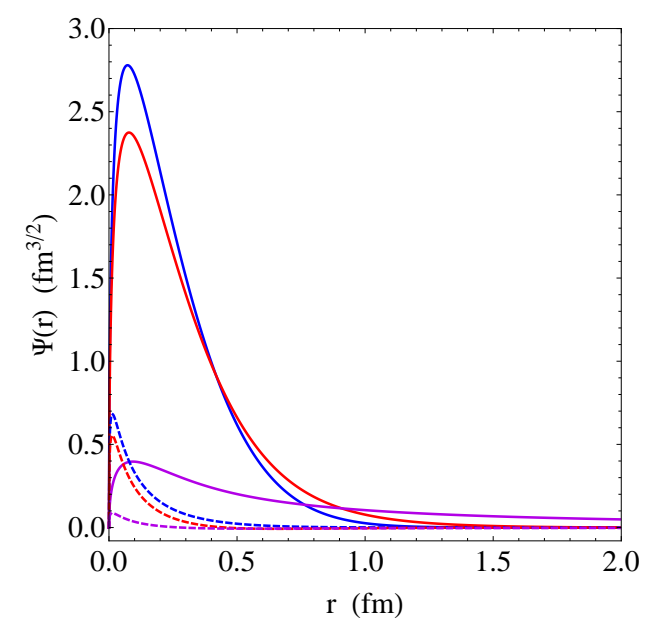

FIG. 1: The $S$-wave (solid lines) and $D$-wave (dashed lines) functions for the $J / \psi$ meson at three temperatures in the limit of quark potential $V=F$. From the top down the temperature $T$ is zero, critical temperature $T_{c}$ and relativistic dissociation temperature $T_{d}$.

The $S-$ and $D$-wave functions for the meson $J / \psi$ at different temperature are shown in Fig 1 in the limit of $V=F$. The wave functions in vacuum are very similar to the results obtained in [13], and the component $1^{3} S_{1}$ dominates the state at any temperature. With increasing temperature, both the $S$ - and $D$-wave functions expand 
continuously. At the critical temperature of deconfinement $T_{c}$, while the peak values of the wave functions drop down a little, their behavior is similar to the one in vacuum. This means that the $J / \psi$ meson is still a bound state at the deconfinement phase transition where the light mesons start to melt in the hot medium, and therefore the observed $J / \psi \mathrm{s}$ in the final state of heavy ion collisions can signal the QGP formation in the early stage. However, with further increasing temperature, the wave functions expand rapidly around $T_{d}=1.35 T_{c}$. By calculating the average distance between the $c$ and $\bar{c}$,

$$
\langle r\rangle(T)=\frac{\int d r r^{3}|\psi(r, T)|^{2}}{\int d r r^{2}|\psi(r, T)|^{2}},
$$

we found that at $T_{d}$ the average size becomes infinite, see Fig 2 , which indicates the $J / \psi$ dissociation. Therefore, $T_{d}$ is called the relativistic dissociation temperature.

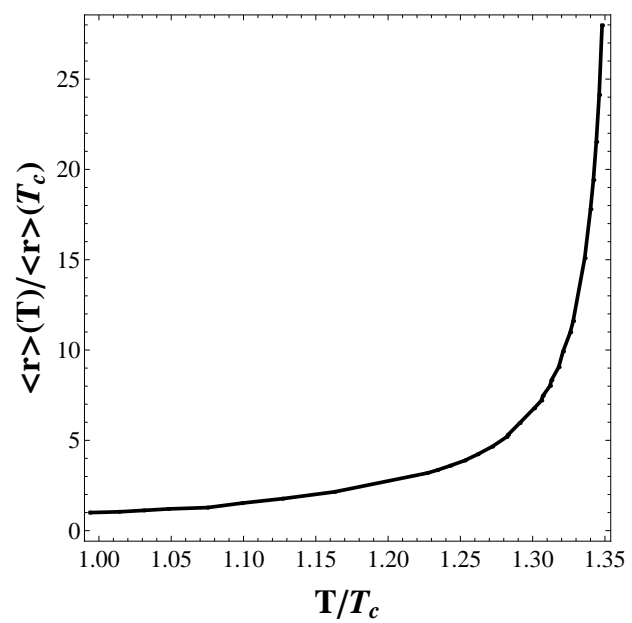

FIG. 2: The average size of the $J / \psi$ meson in the quark-gluon plasma in the limit of potential $V=F$. The temperature and average size are scaled by their corresponding values at the critical point of the deconfinement.

The other quantity which can be used to characterize the quarkonium dissociation is the heavy quark binding energy. For the Dirac equation or equivalently the Schrödinger equations (3) and (4), the usual binding energy defined as $\epsilon=V(\infty)+2 m_{c}-m_{m}$ is no longer valid to describe the quarkonium dissociation. In the limit of $r \rightarrow \infty$, the four equations for the spin singlet and triplet degenerate and the asymptotic equation is simplifies as

$$
\left(-\frac{d^{2}}{d r^{2}}+2 m_{w} V(\infty)+V^{2}(\infty)\right) u=b^{2} u
$$

with $V(\infty)=B(\infty)$, and the energy for the scattering state is

$$
2 m_{w} V(\infty)+V^{2}(\infty)=b^{2}
$$

which leads to the binding energy for the $c \bar{c}$ bound state

$$
\epsilon(T)=V(\infty, T)+\sqrt{V^{2}(\infty, T)+4 m_{c}^{2}}-m_{m}
$$

Fig 3 shows the temperature dependence of the binding energy in the QGP phase and in the limit of potential $V=F$. It drops down monotonously with increasing temperature and reaches zero at $T_{d}$. The result is consistent with the calculation of the charmonium average size. The infinite size and zero binding energy of the $c \bar{c}$ system define the unique dissociation temperature. In comparison with the non-relativistic calculation, the $J / \psi$ dissociation temperature increases from $1.26 T_{c}$ to $1.35 T_{c}$, the relativistic correction is $7 \%$. We also calculated the $J / \psi$ wave functions at finite temperature in the other limit of potential $V=U$ and found that the dissociation temperature $T_{d}$ goes up from the non-relativistic value $2.1 T_{c}$ to $2.38 T_{c}$. The relativistic correction is $13 \%$.

The wave function for the excited state $\chi_{0}$ is shown in Fig 4 in the limit of potential $V=F$. Considering the fact that the radial function $\Psi$ is divergent at the origin [13], we plot $u=r \Psi$ directly from the Schrödinger equation (33). In comparison with the ground state $J / \psi, \chi_{0}$ wave function distributes in a wider region, the average size is larger and the binding energy is smaller. The corresponding dissociation temperature defined by $\langle r\rangle\left(T_{d}\right)=\infty$ and $\epsilon\left(T_{d}\right)=0$ 


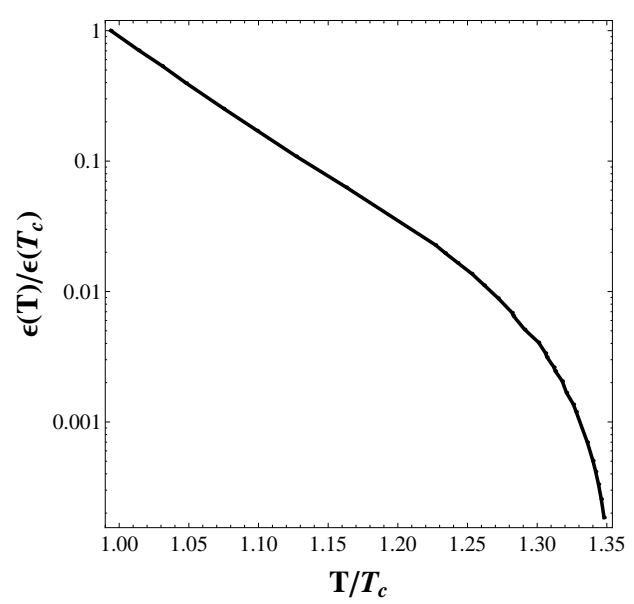

FIG. 3: The binding energy of the $J / \psi$ meson in the quark-gluon plasma and in the limit of potential $V=F$. The temperature and binding energy are scaled by their corresponding values at the critical point of the deconfinement.

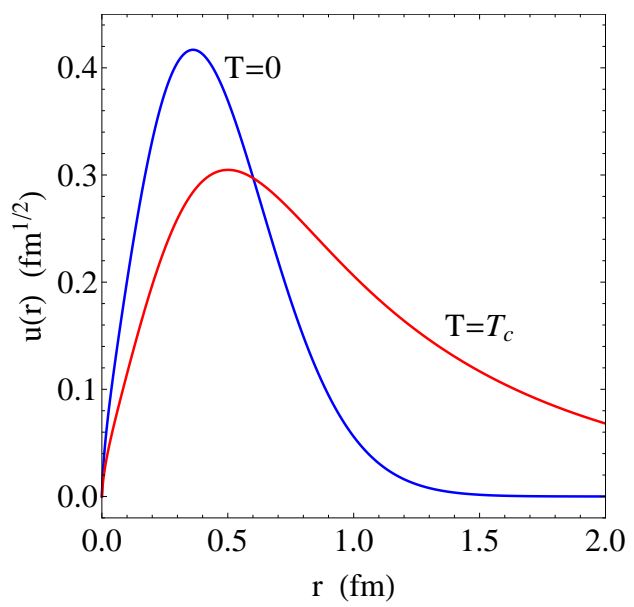

FIG. 4: The wave function $u(r)=r \Psi(r)$ for the $\chi_{0}$ meson at zero temperature and critical temperature of deconfinement $T_{c}$ in the limit of quark potential $V=F$.

is around the critical temperature $T_{c}$ and a little bit higher than the non-relativistic value. It is easy to understand that the relativistic correction for the excited states should be smaller than the one for the ground state.

In summary, we calculated the charmonium wave functions at finite temperature by solving the covariant relativistic Schrödinger equations for the $c \bar{c}$ spin singlet and triplet states. The relativistic effect makes the quark potential well more deep, and charmonia can survive in a more hot medium. By considering the two limits of the central potential, the relativistic correction to the $J / \psi$ dissociation temperature in the QGP phase is in between $7 \%$ and $13 \%$.

Acknowledgement: The work is supported by the NSFC (Grant Nos. 10975084 and 11079024), RFDP (Grant No.20100002110080) and MOST (Grant No.2013CB922000).

[1] J.W.Harris and B.Muller, Annu. Rev. Nucl. Part. S. 46, 71(1996).

[2] T.Matsui and H.Satz, Phys. Lett. B178, 416(1986).

[3] S.Digal, O.Kaczmarek, F.Karsch, and H.Satz, Eur. Phys. J. C43, 71(2005).

[4] O.Kaczmarek, Eur. Phys. J. C61, 811(2009).

[5] H. Satz, J. Phys. G32, R25(2006).

[6] Y.Nambu, Prog. Theor. Phys. 5, 614(1950).

[7] E.E.Salpeter and H.A.Bethe, Phys. Rev.84, 1232 (1951).

[8] E.E.Salpeter, Phys. Rev. 87, 328 (1952). 
[9] I.T.Todorov, Phys. Rev. D3,2351(1971).

[10] P.Long and H.W.Crater, J. Math. Phys. 39, 124(1998).

[11] H.W.Crater, R.L.Becker, C.Y.Wong and P.Van Alstine, Phys. Rev.D 46, 5117 (1992).

[12] H.W.Crater and P.Van Alstine, Phys. Rev. D70, 034026(2004).

[13] H.W.Crater, J.Yoon, and C.Wong, Phys. Rev. D79, 034011(2009).

[14] J.H.Yoon, B.N.Kim, H.W.Crater and C.Y.Wong, arXiv:1110.1598 [hep-ph].

[15] H.W.Crater and C.Y.Wong, J. Phys. Conf. Ser.69,012021 (2007)

[16] H.W.Crater and P.Van Alstine, Phys. Rev. D 37, 1982 (1988).

[17] B.Pan, arXiv:1106.3028 [hep-ph].

[18] H.W.Crater and P.Van Alstine, Phys. Rev. D36: 30007(1987).

[19] E.Shuryak, I.Zahed, Phys. Rev. D70, 054507(2004).

[20] C.Wong, Phys. Rev. C65, 034902(2002); Phys. Rev. C72, 034906(2005).

[21] H.W.Crater, J. Comp. Phys. 115, 470(1994). 